\title{
Sentinel node biopsy in midline tumors: we should be careful and accurate
}

\author{
Sergey Novikov ${ }^{1}$
}

Received: 12 August 2020 / Accepted: 19 August 2020 / Published online: 2 September 2020

(c) The Japanese Society of Nuclear Medicine 2020

Keywords Sentinel lymph-node biopsy · Uterine cervix cancer · Lymphoscintigraphy

First of all, we would like to thank Dr. Ramin Sadeghi for his very important and valuable comments. We completely agree that a hemipelvis approach to biopsy of sentinel lymph nodes (SLNs) for uterine cervix cancer can be very perspective and effective. The efficacy of this algorithm was proposed at the beginning of the century [1], and the recommendation to perform a complete lymphadenectomy in any hemipelvis that does not mapped is widely accepted in clinical practice.

Our personal data are mostly in agreement with this approach: 3 women with unilateral localization of SLNs had metastases in hemipelvis that were not mapped and the last patient had bilateral metastases in non-SLNs.

At the same time, we must also consider another point of view when "the pelvic basin is regarded as a unit" [2]. According to this approach, accuracy and safety of SLN biopsy are sufficiently higher in women with small $(<20 \mathrm{~mm})$ tumors and/or bilateral lymph flow. In the literature, there are enough data supporting this approach. For example, Salvo et al. [3] reported the cases of "negative SLNs detected in one hemipelvis and metastases in nonSLNs in contralateral hemipelvis that did not map". Tanaka et al. [4] observed " 9 cases of metastases in the ipsilateral lymph nodes with no SLN metastasis". Tax et al. [5] in diagnostic review of 43 studies mentioned that they "advise to perform a full pelvic lymph-node dissection whenever the SLNs are not bilaterally detected".

This reply refers to the comment available online at https://doi. org/10.1007/s12149-020-01515-1.

Sergey Novikov

krokon@mail.ru

1 Department of Radiation Oncology and Nuclear Medicine, N.N. Petrov Institute Oncology, Saint Petersburg, Russia
You are right, if we are talking about the "whole truth", we must consider both approaches as possible options. In our practice, we prefer to be more conservative, because we believe that it is safer.

In conclusion, we must underline that we often come across the statement that "the use of lymphoscintigraphy in patients with cervical cancer does not modify the subsequent diagnostic procedure". We are in full agreement with Dr. Ramin Sadeghi that visualization is a very important and probably an obligatory part of SLN biopsy algorithm.

\section{Dr. Sergey Novikov}

Funding None.

\section{References}

1. Plante M, Renaud MC, Têtu B, Harel F, Roy M. Laparoscopic sentinel node mapping in early-stage cervical cancer. Gynecol Oncol. 2003;91(3):494-503.

2. Altgassen C, Hertel H, Brandstädt A, Köhler C, Dürst M, Schneider A, AGO Study Group. Multicenter validation study of the sentinel lymph node concept in cervical cancer: AGO Study Group. J Clin Oncol. 2008;26(18):2943-51.

3. Salvo G, Ramirez PT, Levenback CF, Munsell MF, Euscher ED, Soliman PT, Frumovitz M. Sensitivity and negative predictive value for sentinel lymph node biopsy in women with early-stage cervical cancer. Gynecol Oncol. 2017;145(1):96-101.

4. Tanaka T, Terai Y, Ashihara K, et al. The detection of sentinel lymph nodes in laparoscopic surgery for uterine cervical cancer using 99m-technetium-tin colloid, indocyanine green, and blue dye. J Gynecol Oncol. 2017;28(2):e13. https://doi.org/10.3802/ jgo.2017.28.e13.

5. Tax C, Rovers MM, de Graaf C, Zusterzeel PL, Bekkers $\mathrm{RL}$. The sentinel node procedure in early stage cervical cancer, taking the next step; a diagnostic review. Gynecol Oncol. 2015;139(3):559-67.

Publisher's Note Springer Nature remains neutral with regard to jurisdictional claims in published maps and institutional affiliations. 